\title{
Segmenting a Surface Mesh into Pants Using Morse Theory
}

\author{
Mustafa Hajij ${ }^{\mathrm{b}}$, Tamal Dey ${ }^{\mathrm{a}}, \mathrm{Xin} \mathrm{Li}^{\mathrm{c}}$ \\ ${ }^{a}$ Department of Computer Science, The Ohio State University \\ ${ }^{b}$ Department of Mathematics, University of South Florida \\ ${ }^{c}$ Department of Electrical Engineering, Louisiana State University
}

\begin{abstract}
A pair of pants is a genus zero orientable surface with three boundary components. A pants decomposition of a surface is a finite collection of unordered pairwise disjoint simple closed curves embedded in the surface that decompose the surface into pants. In this paper we present two Morse theory based algorithms for pants decomposition of a surface mesh. Both algorithms operates on a choice of an appropriate Morse function on the surface. The first algorithm uses this Morse function to identify handles that are glued systematically to obtain a pants decomposition. The second algorithm uses the Reeb graph of the Morse function to obtain a pants decomposition. Both algorithms work for surfaces with or without boundaries. Our preliminary implementation of the two algorithms shows that both algorithms run in much less time than an existing state-of-the-art method, and the Reeb graph based algorithm achieves the best time efficiency. Finally, we demonstrate the robustness of our algorithms against noise.
\end{abstract}

Keywords:

Segmentation, Pants Decomposition, Handle Decomposition, Morse Theory, Reeb Graph

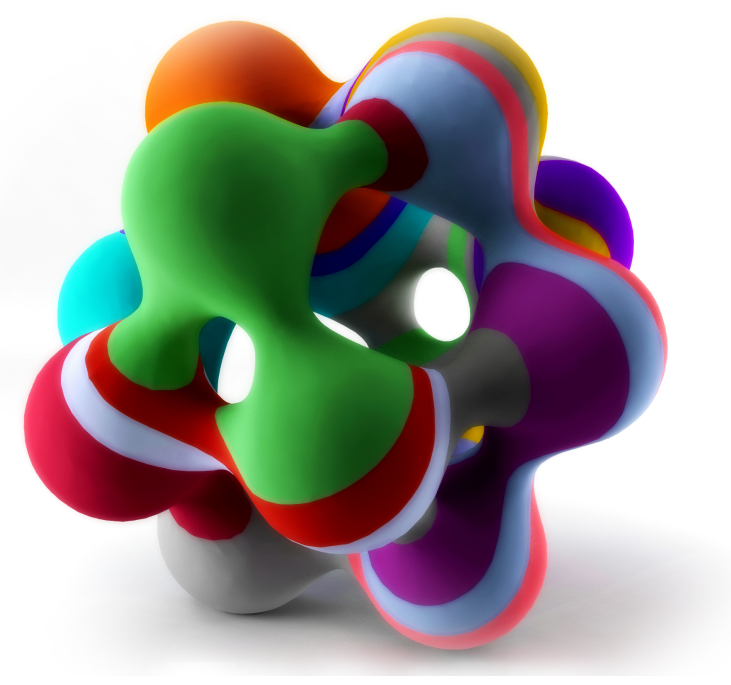

Figure 1: A pants decomposition of a high genus surface by our algorithm.

\section{Introduction}

Segmenting a surface mesh into simple pieces for further processing is a fundamental problem in many mesh processing applications such as texture mapping (Lévy et al., 2002), collision detection ( $\mathrm{Li}$ et al., 2001), skeletonization (Biasotti

Email addresses: mhajij@usf .edu (Mustafa Hajij), tamaldey@cse.ohio-state.edu (Tamal Dey), xinli@cct.lsu.edu (Xin Li)

Preprint submitted to Elsevier et al., 2003) and three-dimensional shape retrieval (Zuckerberger et al., 2002). In surface matching, authors in (Li et al. 2009) showed the use of a particular type of mesh segmentation called a pant decomposition, see Figure 1. It derives its name from its constituent piece termed a pair of pants, which, up to topology, is a genus zero orientable surface with 3 boundary components. Besides surface matching, pants decomposition has found applications in surface classification and indexing (Jin et al., 2009), and consistent mesh parametrization (Kwok et al. 2012). This type of segmentation can be viewed as a common base domain segmentation (Kwok et al., 2012), where one partitions the mesh into parts with a common property such as having the same topology. Authors in (Li et al. 2009) presented a pant decomposition algorithm based on the computations of certain basis cycles in the first homology group of the input surface. Other pants decomposition related algorithms proposed in the graphics literature can be found in (Zhang and Li 2012) where the authors enumerates different classes of pants decompositions. However, these methods rely on computing certain curves on the surface called Handle and Tunnel loops (Dey et al. 2007, 2013). Computing such curves is expensive and more importantly requires the surface to be embedded in $\mathbb{R}^{3}$ and not have any boundary. Morse theory allows our algorithms to get rid of these two constraints. Moreover, pants decomposition relies on finding a collection curves on the surface with certain topological properties. Finding these curves and manipulating them is a difficult problem. Morse theory allows us to avoid defining these curves explicitly by realizing them implicitly as level curves of a Morse function.

Many segmentation algorithms have been proposed in the graphics literature includes (Mangan and Whitaker, 1999,

September 8, 2016 
Shamir, 2008; Li et al., 2009; Chen et al., 2009, Shlafman et al., 2002; Attene et al., 2006a; Yu and Li, 2015; Li et al., 2016). One reason for the variety of segmentation algorithms suggested in the literature is that there is no one universal good algorithm that suits all applications. The techniques used in the algorithms are related to other areas in computer graphics such as image segmentation (Reynolds et al., 1995; Tomasi and Manduchi 1998) and machine learning (Karypis and Kumar, 1998, Cover and Hart, 1967). For good surveys on various segmentation algorithms see (Attene et al., 2006b; Shamir, 2008).

In this work we propose two algorithms based on Morse theory for computing a pant decomposition of an input surface mesh. Morse theory connects the geometry and topology of manifolds via the critical values/points of a specific class of real-valued functions called Morse functions. Intuitively, given such a function $f$ on a manifold $M$, Morse theory studies the topological changes of the level sets of $f$. These level sets change in topology only at the critical values. Consequently, the space in between two consecutive critical levels becomes a product space of a fiber with an interval. This fact is used to decompose a surface into pants and cylinders, and the latter parts are glued inductively into pants. The second algorithm exploits a reduced structure called Reeb graph (Reeb, 1946) derived from a function $f$ on the surface. The Reeb graph of $f$ is a quotient space derived from $M$ and $f$. For a Morse function, all vertices in the Reeb graph have valence 3 except the extrema. We exploit this property to compute a pair of pant for every degree-3 vertex of the Reeb graph and treat the extrema specially.

Morse theory, in its original form, assumes smooth settings (Milnor, 1963). For operating on surface meshes, we need a piecewise linear (PL) version of the theory. We make this transition using the PL Morse theory proposed by (Banchoff et al. 1967). We show how one can compute a function on a surface mesh satisfying the specific property that our algorithms require. Furthermore, we extend our basic algorithms to the case when the surface has boundaries, or when the function $f$ allows degenerate critical points such as monkey saddles.

Our experiments show that both of our algorithms run much faster in practice than the algorithm of Li et al. while the Reebgraph based algorithm performs the best. Furthermore, our tests show that both of the algorithms presented here are robust to noise.

\section{Morse Theory and Handle Decomposition for Surfaces}

Let $M$ be a compact smooth surface, and let $I=[a, b] \subseteq \mathbb{R}$, where $a<b$, be a closed interval. Let $f: M \longrightarrow I$ be a smooth function defined on $M$. A point $x \in M$ is called a critical point of $f$ if the differential $d f_{x}$ is zero. A value $c$ in $\mathbb{R}$ is called a critical value of $f$ if $f^{-1}(c)$ contains a critical point of $f$. A point in $M$ is called a regular point if it is not a critical point. Similarly, a value $c \in \mathbb{R}$ is called regular if it is not critical. The inverse function theorem implies that for every regular value $c$ in $\mathbb{R}$ the level set $f^{-1}(c)$ is a 1-manifold, i.e., $f^{-1}(c)$ is a disjoint union of simple closed curves. A critical point is called non- degenerate if the matrix of the second partial derivatives of $f$, called the Hessian matrix, is non-singular.

The definition of a Morse function is motivated mainly by the following Lemma.

Lemma 1. (Morse Lemma) Let $M$ be a smooth surface, $f: M \longrightarrow I$ be a smooth function and $p$ be a non-degenerate critical point of $f$. We can choose a chart $(\phi, U)$ around $p$ such that $f \circ \phi^{-1}$ takes exactly one of the following three forms:

$$
\begin{aligned}
& \text { 1. } f \circ \phi^{-1}(X, Y)=X^{2}+Y^{2}+c . \\
& \text { 2. } f \circ \phi^{-1}(X, Y)=-X^{2}-Y^{2}+c . \\
& \text { 3. } f \circ \phi^{-1}(X, Y)=X^{2}-Y^{2}+c .
\end{aligned}
$$

The index of a critical point $x$ of $f$, denoted by index $f(x)$, is defined to be the number of negative eigenvalues of its Hessian matrix. Since the Hessian of a scalar function on smooth surface is a $2 \times 2$ symmetric matrix, then the index takes the values 0,1 or 2 . One can see that on a non-degenerate critical point of index 0 the function $f$ takes a minimum value, on a non-degenerate critical point of index 1 , the graph of the function looks like a saddle and on a non-degenerate critical point of index 2 the function $f$ takes a maximum value. See Figure 2
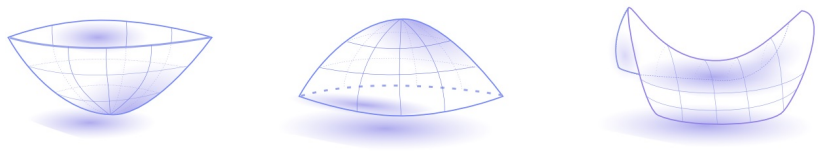

Figure 2: Minimum, Maximum, and Saddle.

If all the critical points of $f$ are non-degenerate and all critical points have distinct values, then $f$ is called a Morse function. If the surface $M$ has boundary, then we also require two other conditions : (1) $f^{-1}(\partial I)=\partial M$ and (2) the critical points of $f$ lie in the interior of $M$.

\subsection{Handle Decomposition of a Surface}

Our first algorithm uses the attachment of cylinders called handles at the critical points. We need a few definitions first.

Let $M$ be a smooth surface and let $f: M \longrightarrow \mathbb{R}$ be a Morse function defined on $M$. Define the set

$$
M_{f, t}=\{x \in M: f(x) \leq t\} .
$$

Let $a, b, a<b$, be two reals. Define

$$
M_{f,[a, b]}=\{x \in M: a \leq f(x) \leq b\} .
$$

When it is clear from the context, we will drop $f$ from the notation and use simply $M_{t}$ and $M_{[a, b]}$ to refer to the previous two sets. Morse theory studies the topological changes of $M_{t}$ as $t$ varies. The following is well-known (Matsumoto, 2002).

Theorem 2. Let $f: M \longrightarrow \mathbb{R}$ be a smooth function on a smooth surface $M$. For two reals $a, b, a<b$, if $f$ has no critical values in the interval $[a, b]$, then the surfaces $M_{a}$ and $M_{b}$ are diffeomorphic. 
The previous theorem says that the topology of the surface $M_{t}$ does not change as $t$ passes through regular values. In the following we use $D^{1}$ to denote the interval $[0,1]$. The end points of $D^{1}$ are given by $\partial D^{1}=\{0,1\}$. Given a Morse function $f$ on $\mathrm{M}$, the following theorem gives a precise description for the change that occurs in the topology of $M_{t}$ as $t$ passes through a critical value.

Theorem 3. Let $f: M \longrightarrow \mathbb{R}$ be Morse function. Let $p$ be a critical point of index $i$ and $f(p)=t$ be its corresponding critical value. Let $\epsilon$ be chosen small enough so that $f$ has no critical values in the interval $[t-\epsilon, t+\epsilon]$.

1. If index $x_{f}(p)=0$, then $M_{t+\epsilon}$ is diffeomorphic to the disjoint union of $M_{t-\epsilon}$ and a 2-dimensional disk $D^{2}$.

2. If index $f(p)=1$, then $M_{t+\epsilon}$ can be obtained from $M_{t-\epsilon}$ by attaching a 1-handle. This means that $M_{t+\epsilon}$ can be obtained by gluing a rectangular strip $D^{1} \times D^{1}$ to the boundary of $M_{t-\epsilon}$ along $D^{1} \times \partial D^{1}$.

3. If index $(p)=2$, then $M_{t+\epsilon}$ can be obtained by capping off the surface $M_{t-\epsilon}$ with a disk $D^{2}$. This means that $M_{t+\epsilon}$ is obtained by gluing a disk $D^{2}$ along its boundary $\partial D^{2}$ to one of the boundary components of $M_{t-\epsilon}$.

\subsection{Morse Theory for Triangulated Surfaces}

Morse theory was extended to triangulated surfaces (2manifolds) by Banchoff (Banchoff et al., 1967). Recently, Morse theory has found applications in global surface parameterization Guo et al. (2006), finding a fundamental domain of a surface $\mathrm{Ni}$ et al. (2004), surface quadrangulation Dong et al. (2006), topological matching Hilaga et al. (2001), implicit surfaces Stander and Hart (1997), surface segmentation Yamazaki et al. (2006), spline construction Wang et al. (2009) and many other applications.

Let $M$ be a triangulated surface, and let $f: M \longrightarrow I$ be a piece-wise linear (PL) continuous function on $M$. The link $L k(v)$ of a vertex $v$ is defined as the set of all vertices $w$ that $v$ shares an edge $[v, w]$ with. The upper link of $v$ is defined as

$$
L k^{+}(v)=\{u \in L k(v): f(u)>f(v)\}
$$

and the lower link is defined by

$$
\operatorname{Lk}^{-}(v)=\{u \in \operatorname{Lk}(v): f(u)<f(v)\}
$$

and mixed link

$$
L k^{ \pm}(v)=\left\{\left(u_{1}, u_{2}\right): f\left(u_{1}\right)<f(v)<f\left(u_{2}\right)\right\} .
$$
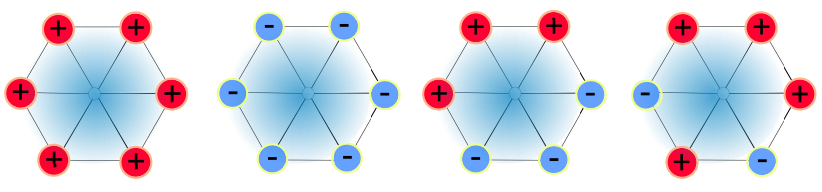

Figure 3: (a) minimum, (b) maximum, (c) regular vertex, (d) saddle .
Using the link definitions we can classify the interior vertices of $M$. The boundary vertices are treated separately as we will require $f$ to have identical values on them. An interior vertex $v$ is regular if $\left|L k^{ \pm}(v)\right|=2$, is a maximum with index 2 if $\left|L k^{+}(v)\right|=0$, is a minimum with index 0 if $\left|L k^{-}(v)\right|=0$, and is a saddle with index 1 and multiplicity $m \geq 1$ if $\left|L k^{ \pm(v)}\right|=2+2 m$. See Figure 3. A vertex is a simple critical point if it is either minimum, or maximum, or a saddle with multiplicity 1 . A PL function on a closed triangulated surface $M$ is PL Morse if all its vertices are either PL regular or simple PL critical and have distinct function values. If the mesh $M$ has a non-empty boundary then we also require (1) $f^{-1}(\partial I)=\partial M$, and (2) the critical vertices of $f$ lie in the interior of $M$.

\subsection{Reeb Graph}

Let $M$ be a surface possibly with boundary $\partial M$, and let $f: M \longrightarrow[0,1]$ be continuous. Define the equivalence relation $\sim$ on $M$ by $x \sim y$ if and only if $f(x)=f(y)=c \in[0,1]$ and $x$ and $y$ belong to the same connected component of the level set $f^{-1}(c)$. The set $R(f)=X / \sim$ with the standard quotient topology is called Reeb graph of $f$. When $f$ is smooth and Morse, or PL Morse, every vertex of the $R(f)$ arises from a critical point of $f$ or a boundary component. Every maximum or minimum of $f$ gives rise to a degree 1-node of $R(f)$. Since $f$ is Morse, every boundary component also gives rise to a degree 1 -node and every saddle of $f$ gives rise to degree 3-node. If $M$ is an embedded surface without boundary then $M$ can be recovered up to a homeomorphism from $R(f)$ as the boundary of an oriented 3-dimensional regular neighborhood of the graph $R(f)$ Hatcher and Thurston (1980). This fact is the essence of the Reeb graph-based pant decomposition algorithm 5. See Figure 4 for an example of a Reeb graph.
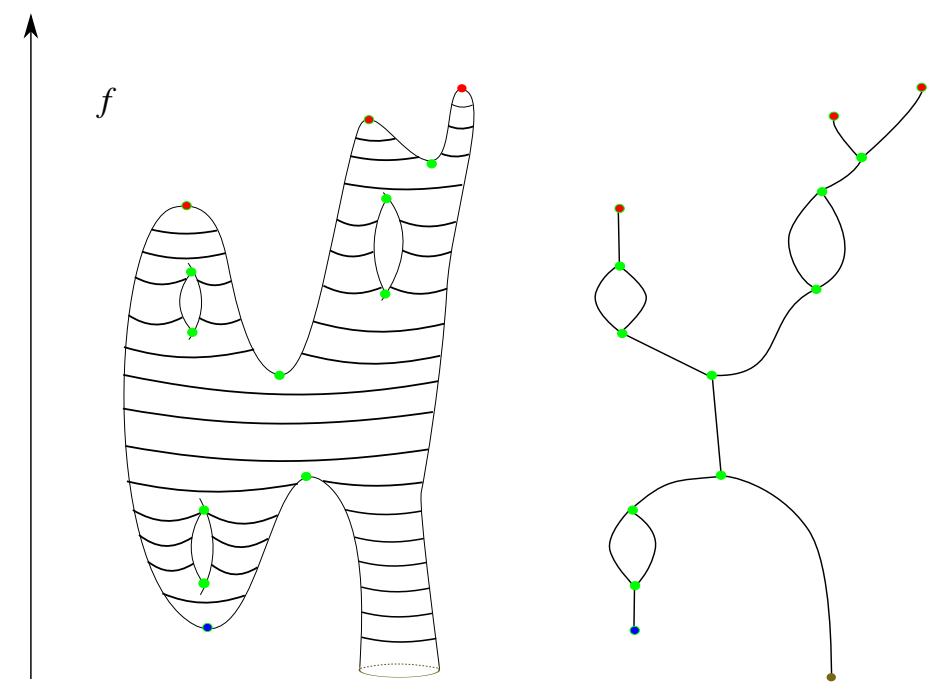

Figure 4: An example of a Reeb graph.

Remark 1. One reason that we require the Morse function to assume constant values on boundaries is that it makes it consistent with the definition of the Reeb graph we give here. Note that each boundary component maps exactly to one point on the Reeb graph. 
The definition of Reeb graph goes back to G. Reeb (Reeb. 1946). It was first introduced to computer graphics in (Sato et al. (1994). Reeb graph has found applications in shape understanding (Attene et al. 2003), quadrangulation (Hétroy and Attali. 2003), surface understanding (Biasotti et al. 2000), segmentation (Werghi et al. 2006), parametrization (Patane et al. 2004, Zhang et al. 2005), animation (Kanongchaiyos and Shinagawa, 2000) and many other applications. Reeb graphs algorithms can be found in many papers such as (Shinagawa and Kunii, 1991; Cole-McLaughlin et al., 2003; Pascucci et al. 2007; Doraiswamy and Natarajan, 2009). The most efficient algorithms in terms of time complexity are due to (Parsa, 2012. Harvey et al., 2010).

\section{Pants Decomposition}

Let $M$ be a compact, orientable, and connected surface. We say that $M$ is of type $(g, b)$ if $M$ is of genus $g$ and has a $b$ boundary components. A pair of pants is a surface of type $(0,3)$. See Figure 5 .

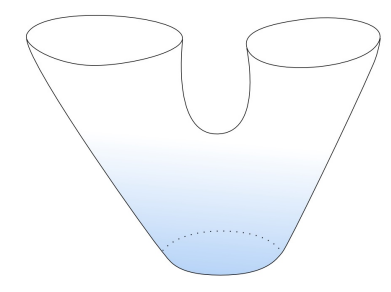

Figure 5: A pair of pants

A pants decomposition of $M$ is a finite collection of unordered pairwise disjoint simple closed curves $\left\{c_{1}, \ldots c_{n}\right\}$ embedded in $M$ with the property that the closure of each connected component of $M-\left(c_{1} \cup \ldots \cup c_{n}\right)$ is a pair of pants. Two pants decompositions of $M$ are equivalent if they are isotopic. More precisely, we two pants decompositions $\left\{\alpha_{1}, \ldots \alpha_{n}\right\}$ and $\left\{\beta_{1}, \ldots \beta_{n}\right\}$ of a surface $M$ are equivalent if there exists a homotopy

$$
H: S^{1} \times[0,1] \longrightarrow M
$$

that takes the curves $\left\{\alpha_{1}, \ldots \alpha_{n}\right\}$ to the curves $\left\{\beta_{1}, \ldots \beta_{n}\right\}$ such that for every $t \in[0,1]$ the map $H\left(S^{1} \times\{t\}\right)$ is a homemorphism onto the image. Here $S^{1}$ denotes the unit circle. See Figure 19 for an example of two non-isotopic pants decompositions of a genus-2 surface.
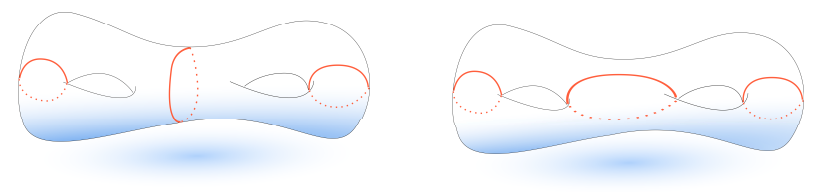

Figure 6: Two non-isotopic pants decompositions of a genus 2 surface.

Let $M$ be a compact orientable surface and connected surface of type $(g, b)$. The Euler characteristic of $\mathrm{M}$, denoted by $\chi(M)$ is defined as $\chi(M)=2-2 g-b$. Every connected, compact and orientable surface $M$ with $\chi(M)<0$, genus $g$ and $b$ boundary components admits a pants decomposition with $3 g-3+b$ simple closed curves and the number of complementary components is $2 g-2+b=|\chi(M)|$.

\section{Handle-Based Pants Decomposition}

In this section we use handles given by a Morse function to design an algorithm for decomposing a surface $M$ with $\chi(M)<0$ into a collection of surfaces of type $(0,3)$. Our algorithm works for arbitrary surface $M$ with $\chi(M)<0$ and with or without a boundary. However, in order to guarantee the correctness of our algorithm we must choose a function with certain properties. Ideally, this function should be PL Morse with these properties when $M$ is a surface mesh. We cannot always guarantee that the function is PL Morse, but we can compute one that satisfies all required properties except the simplicity of the saddles. We first describe the algorithm assuming a PL Morse function and later mention how to handle the exceptions of degenerate saddles.

\subsection{Orientable Surfaces With $\chi(\cdot)<0$ and No Boundary}

In this section we give an algorithm to compute a pants decomposition of a triangulated surface with genus $g \geq 2$ without boundary. Let $M$ be a compact connected orientable surface with genus $g \geq 2$ without boundary and let $f$ be a PL Morse function on $M$. Suppose that $t_{1}, t_{2}, \ldots, t_{n}$ are the critical values for $f$ ordered in an ascending order. Let $p_{1}, p_{2}, \ldots, p_{n}$ be the corresponding critical points of $f$. Choose a real number $\epsilon>0$ small enough so that for each $1 \leq i \leq n$ there are no critical values for $f$ on the interval $\left[t_{i}-\epsilon, t_{i}+\epsilon\right]$ except $t_{i}$. Finally we assume that function $f$ has exactly one minimum and exactly one maximum. It is clear from the choice of the function $f$ that one of the points $p_{1}$ and $p_{n}$ is the global maximum and one of them is the global minimum. Since multiplying any Morse function on a surface by -1 changes its critical points of index 0 to critical points of index 2 and vice versa, we can always choose our Morse function $f$ so that $p_{1}$ is the global minimum and $p_{n}$ is the global maximum. It should be noted that such a function can be constructed in practice and we will talk about the construction of such functions later. We need the following lemma for the correctness of our algorithm.

Lemma 4. Let $M$ be a compact connected orientable surface with a Morse function $f$ chosen as specified above then $M_{t_{3}+\epsilon}$ is homeomorphic to a surface of type $(1,1)$ or a surface of type $(0,3)$.

Proof. The choice of the scalar function $f$ implies immediately that for each $2 \leq i \leq n-1$ we have $\operatorname{index}_{f}\left(p_{i}\right)=1$. Moreover, by construction we have $\operatorname{index}_{f}\left(p_{1}\right)=0$ and $\operatorname{index}_{f}\left(p_{n}\right)=2$. By Theorem 3 we conclude that $M_{t_{1}+\epsilon}$ is diffeomorphic to a disk and $M_{t_{2}+\epsilon}$ is diffeomorphic to surface of type $(0,2)$.

Moreover, by Theorem 3, when $f$ passes through $t_{3}$ the surface $M_{t_{3}+\epsilon}$ is obtained from $M_{t_{2}+\epsilon}$ by gluing a rectangular strip $D^{1} \times D^{1}$ to the boundary of $M_{t_{2}+\epsilon}$ along $D^{1} \times \partial D^{1}$. Up to a homeomophism, there are two possible ways of gluing the rectangular strip $D^{1} \times D^{1}$ to the boundary of $M_{t_{2}+\epsilon}$ along $D^{1} \times \partial D^{1}$. 

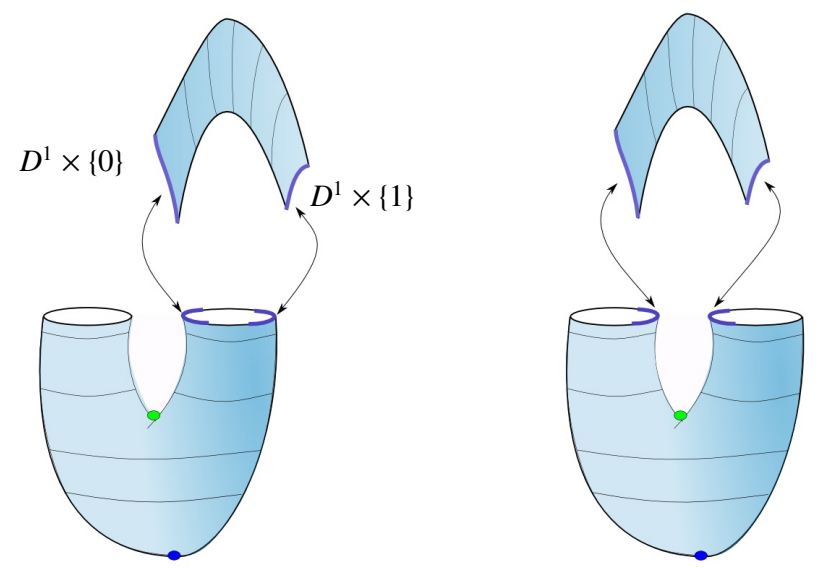

Figure 7: Two possible ways to glue a disk to the surface of type $(0,2)$.

See Figure 7. We either glue this rectangular strip to the same boundary component of $M_{t_{2}+\epsilon}$ to obtain a surface of type $(0,3)$ or we glue each side of the strip on one of the boundary components to obtain a surface of type $(1,1)$. See Figure 8
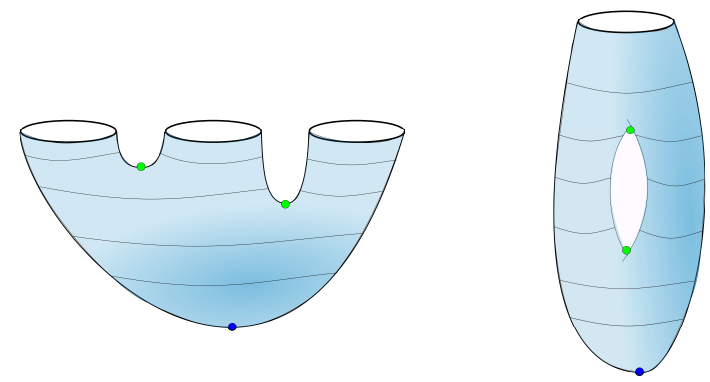

Figure 8: $M_{f, t_{3}+\epsilon}$ is homeomorphic to a type $(0,3)$ or to a type $(1,1)$

Remark 2. Using similar argument one can prove that the surface $M_{t_{n-2}-\epsilon}$ is either of type $(0,3)$ or a surface of type $(1,1)$.

Lemma 4 holds in the case when the function $f$ is PL Morse on a triangulated surface. However, the case when the PL scalar function has saddle points with multiplicity larger than or equal to 2 needs special treatment and Lemma 4 is no longer valid. We deal with such cases in section 4.3

Lemma 4 and remark 2 will be used to obtain the first and the last pants in our pants decomposition. The algorithm is as follows:

1. Compute the critical points of $f$ and put them in an ascending order. Let $p_{1}, p_{2}, \ldots, p_{n}$ be the sequence of ordered critical points of $f$ and let $t_{1}, t_{2}, \ldots, t_{n}$ be their corresponding critical values. Note that $n=2 g+2$ by our choice of the scalar function $f$.

2. For each $3 \leq i<n-3$ let $c_{i}=\frac{t_{i}+t_{i+1}}{2}$. After reindexing, we define the set $C=\left\{c_{i} \mid 1 \leq i \leq 2 g-3\right\}$. In other words, the set $C$ is a set of ordered regular values for $f$ such that there is exactly one critical value for the function $f$ in the intervals $\left[c_{i}, c_{i+1}\right]$ for $1 \leq i \leq 2 g-3$. See Figure 9 for an example.

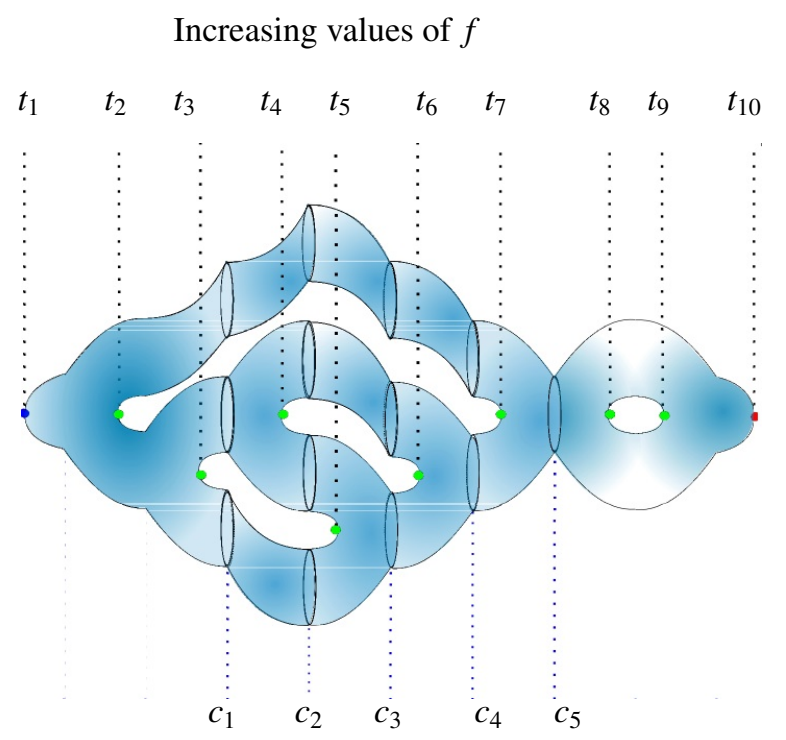

Figure 9: Cutting a surface of genus 4 along the values $c_{i}$.

3. Cut the surface $M$ along the level sets $f^{-1}(c)$ for all $c \in C$. Note again that the values $c \in C$ are all regular values.

4. By Lemma 4 the surface $M_{c_{1}}$ is either of type $(1,1)$ or of type $(0,3)$. If $M_{c_{1}}$ is of type $(1,1)$, then we trace a loop from the saddle point $p_{2}$ to the minimum point $p_{1}$ and cut the surface along that loop. We explain later a method of tracing this loop. See Figure 10 Otherwise, if the surface is of type $(0,3)$ we do not need to do anything. In either cases, we denote the resulting surface of type $(0,3)$ by $M_{\text {initial }}$.

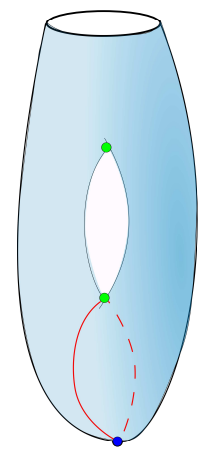

Figure 10: Tracing a loop from the first saddle point $p_{2}$ to the minimum $p_{1}$.

5. Consider the manifold with boundary $M_{\left[c_{1}, c_{2}\right]}$. This surface is a finite disjoint union of one surface of type $(0,3)$ and multiple surfaces of type $(0,2)$. Attach every surface of type $(0,2)$ to $M_{\text {initial }}$. Note that this gluing does not change the homeomorphism type of $M_{\text {initial }}$. See Figure 11 (b). 
6. For each $1<i<2 g-3$ consider the manifold with boundary $M_{\left[c_{i}, c_{i+1}\right]}$. This surface is again a finite disjoint union of one surface of type $(0,3)$ and multiple surfaces of type $(0,2)$. Attach every surface of type $(0,2)$ to $M_{\left[c_{i-1}, c_{i}\right]}$. See Figure $11(\mathrm{c})$.

(a)

(c)

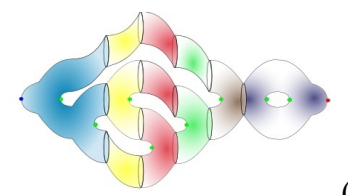

(d)
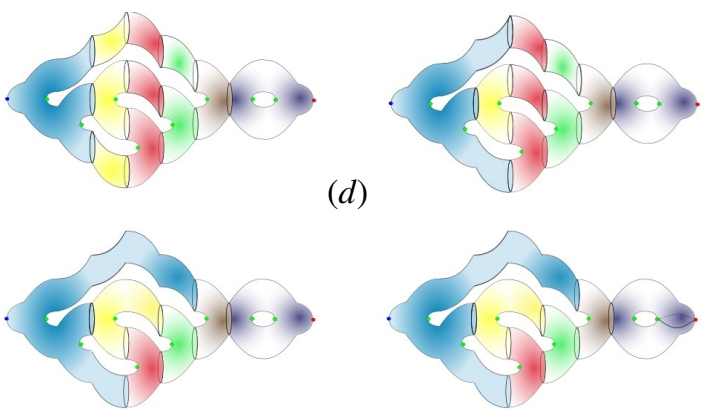

Figure 11: Illustration of the handle-based pants decomposition algorithm : (a) Cutting the surface along $c_{1}, c_{2}, c_{3}, c_{4}$ and $c_{5}$. Note that $M_{\text {initial }}$ is a pants in this example. (b) Attaching cylinders that appear on the second level to $M_{\text {initial }}$ and keeping the pant component. (c) Attaching cylinders at each level to the previous level and keeping the pant component. (d) The surface $M_{-f, c_{2 g-3}}=$ $M_{-f, c_{4}}$ is a surface of type $(1,1)$ so we trace a loop from the saddle to the minimum of $-f$ and cut the surface along this loop.

7. The remaining part $M_{-f, c_{2 g-3}}$ is either of type $(1,1)$ or of type $(0,3)$. If the surface $M_{-f, c_{2 g-3}}$ is of type $(1,1)$ then trace a loop from the saddle point $p_{n-1}$ to the point $p_{n}$ and cut the surface $M_{-f, c_{2 g-3}}$ along this loop to obtain a pant. Otherwise, if the surface is of type $(0,3)$ we do not need to do anything.

Note that the algorithm constructs a collection of pants inductively. We start by having the first pair of pants in step 4 and then we go to the next level which is a finite disjoint union of a single pant and some topological cylinders. We attach the cylinders to the previous pant and then we go to the next level and repeat the same process.

We should clarify here what we mean by tracing a loop from the saddle to the minimum point we mentioned in step (4). Let $p_{2}$ the simple saddle point and $p_{1}$ be the unique minimum point for the PL scalar function $f$ on $M$ specified in our algorithm above. The point $p_{2}$ is a simple saddle, hence the set $L k^{-}\left(p_{2}\right)$ can be decomposed into two disjoint connected components $A$ and $B$. Pick the vertex $v_{A}$ in $A$ such that $f\left(v_{A}\right) \leq f(v)$ for all $v \in A$. The vertex $v_{B}$ is chosen similarly. A descending path from a regular vertex $v_{0}$, denoted by $\operatorname{dpath}\left(v_{0}\right)$, is defined to be a finite sequence of vertices $\left\{v_{0}, \ldots, v_{k}\right\}$ on $M$ such that $\left\langle v_{i}, v_{i+1}>\right.$ is an edge on $M$ for $0 \leq i \leq k-1, f\left(v_{i}\right)<f\left(v_{i-1}\right)$, and $v_{k}$ is a minimum. A loop connecting $p_{2}$ and $p_{1}$ can be computed as the concatenation of $\left.\operatorname{dpath}\left(v_{A}\right),<p_{2}, v_{A}\right\rangle,\left\langle p_{2}, v_{B}\right\rangle$, and $\operatorname{dpath}\left(V_{B}\right)$. The loop in step (7) is computed analogously by considering an ascending path.

\subsection{Orientable Surfaces With $\chi(\cdot)<0$ and Non-Empty Bound- ary}

In this section we present an algorithm to decompose a surface $M$ with $\chi(M)<0$ with boundary components. The algorithm follows almost similarly as before. The main difference is that we need to take care of the choice of the Morse function. We have two cases, (1) The surface $M$ has exactly one boundary component (2) The surface $M$ has more than one boundary component.

Let $M$ be a compact connected orientable surface with $\chi(M)<0$ and one boundary component $\Sigma$. We pick a point $p_{\max }$ on the surface and construct a Morse function $f: M \longrightarrow[0,1]$ that satisfies the following conditions :

1. $f^{-1}(\Sigma)=0$ and $f(x)>0$ for all $x$ in $M \backslash \Sigma$.

2. The point $f^{-1}(1)=x_{\max }$ is a global maximum.

3. The function $f$ does not have any critical point of index 0 or 2 except for $p_{\max }$.

The pants decomposition algorithm for a surface $M$ with $\chi(M)<0$ and one boundary component $\Sigma$ algorithm goes now as follows :

1. Compute the critical points of $f$ and put them in an ascending order. Let $p_{1}, p_{2}, \ldots, p_{n}$ be the ordered critical points of $f$ and let $t_{1}, t_{2}, \ldots, t_{n}$ be the corresponding critical values. By our choice of the Morse function, we have $\operatorname{index}\left(p_{i}\right)=1$ for all $1 \leq i \leq n-1$ and $\operatorname{index}\left(p_{\max }\right)=2$.

2. For each $1 \leq i<n-3$ let $c_{i}=\frac{t_{i}+t_{i+1}}{2}$. Define the set $C=\left\{c_{i} \mid 1 \leq i \leq 2 g-3\right\}$. In other words, the set $C$ is a set of ordered regular values for $f$ such that there is exactly one critical value in the interval $\left[c_{i}, c_{i+1}\right]$. See Figure 12 for an example.

3. Cut the surface $M$ along the level sets $f^{-1}(c)$ for all $c \in C$.

4. Consider the manifold $M_{\text {initial }}:=M_{\left[-\epsilon, c_{1}\right]}$. By our choice of the Morse function $M_{\text {initial }}$ is a of type $(0,3)$.
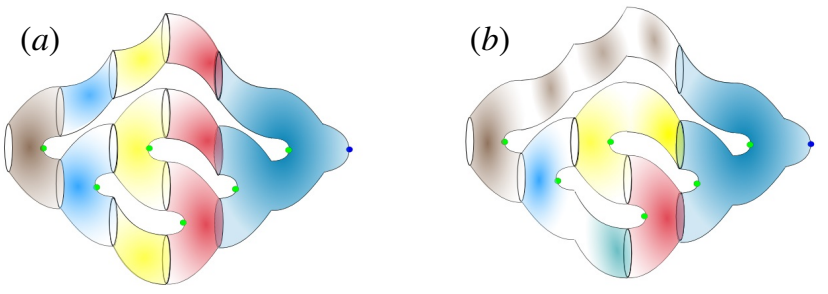

Figure 12: Illustration of the handle-based pants decomposition algorithm on a surface with a single boundary components : (a) Cutting the surface along $c_{1}$, $c_{2}$. $c_{3}$ and $c_{4}$. (b) Attaching cylinders at each level to the previous level and keeping the pants components.

The rest of the pants decomposition algorithm for a surface is similar to steps (5), (6) and (7) of the algorithm in section 4.1. 
Now we discuss the final case. Suppose that $M$ has boundary components $\Sigma_{1}, \ldots, \Sigma_{k}$ where $k \geq 2$. Here we also need to construct a Morse function that serves our purpose. We need a Morse function $f: M \longrightarrow[0,1]$ that satisfies the following :

1. We choose one of the boundary component, say $\Sigma_{1}$, and we construct $f$ such that $f^{-1}(0)=\Sigma_{1}$ and $f(x)>0$ for all $x \in M \backslash \Sigma_{1}$.

2. $f^{-1}(1)=\cup_{i=2}^{k} \Sigma_{i}$.

3. The function $f$ does not have any critical point of index 0 or 2 .

As we did earlier, let $p_{1}, p_{2}, \ldots, p_{n}$ be the ordered critical points of $f$ and let $t_{1}, t_{2}, \ldots, t_{n}$ be the corresponding critical values of $f$. Define the values $c_{i}=\frac{t_{i}+t_{i+1}}{2}$ for all $1 \leq t_{i} \leq n-1$. By our choice of the Morse function, the manifold $M_{\left[c_{i}, c_{i+1}\right]}$ is homeomorphic to a finite disjoint union of one surface of type $(0,3)$ and multiple surfaces of type $(0,2)$. In particular $M_{\left[-\epsilon, c_{1}\right]}$ is a pants. The pants decomposition algorithm now is similar to the previous algorithm except that we do not trace any loop in this case since all connected components after cutting along the regular values of $C$ are pants or cylinders. See Figure 13 for an example.
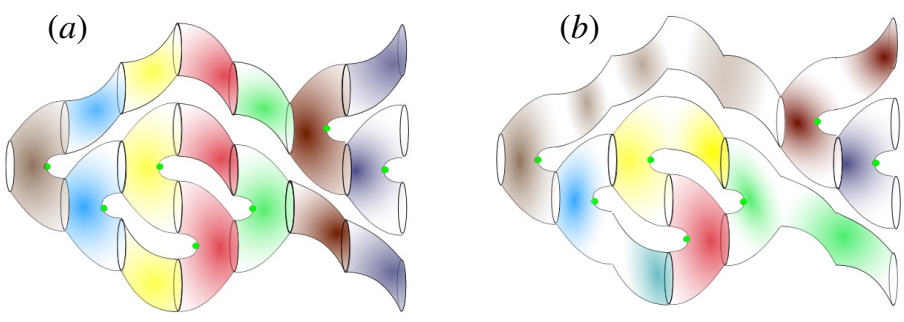

Figure 13: Illustration of the handle-based pants decomposition algorithm on a surface wit multiple boundary components : (a) Cutting the surface along $c_{1}$, $c_{2} . c_{3}, c_{4}, c_{5}$ and $c_{6}$. (b) Attaching cylinders at each level to the previous level and keeping the pants components.

Figure 14 shows multiple examples of pants decomposition of surfaces using this algorithm.

\subsection{Dealing with Degenerate Cases}

In this section we deal with the case when the index of a critical point point $p_{i}$ is 1 and has multiplicity $m \geq 2$. We formulate our arguments in terms of Reeb graph for clarity.

1. If the point $p_{1}$ is a degenerate saddle point then the Reeb graph obtained from the quotient space of $M_{t_{2}+\epsilon}$ appears as in Figure 15

In this case the surface $M_{t_{2}+\epsilon}$ is of type $(0, b)$ where $b>3$ and we can decompose this surface into pants as described earlier.

2. The point $p_{2}$ is a simple saddle and the point $p_{3}$ is a degenerate one. In this case the Reeb graph obtained from the surface $M_{t_{3}+\epsilon}$ has two possibilities and they both appear in Figure 16
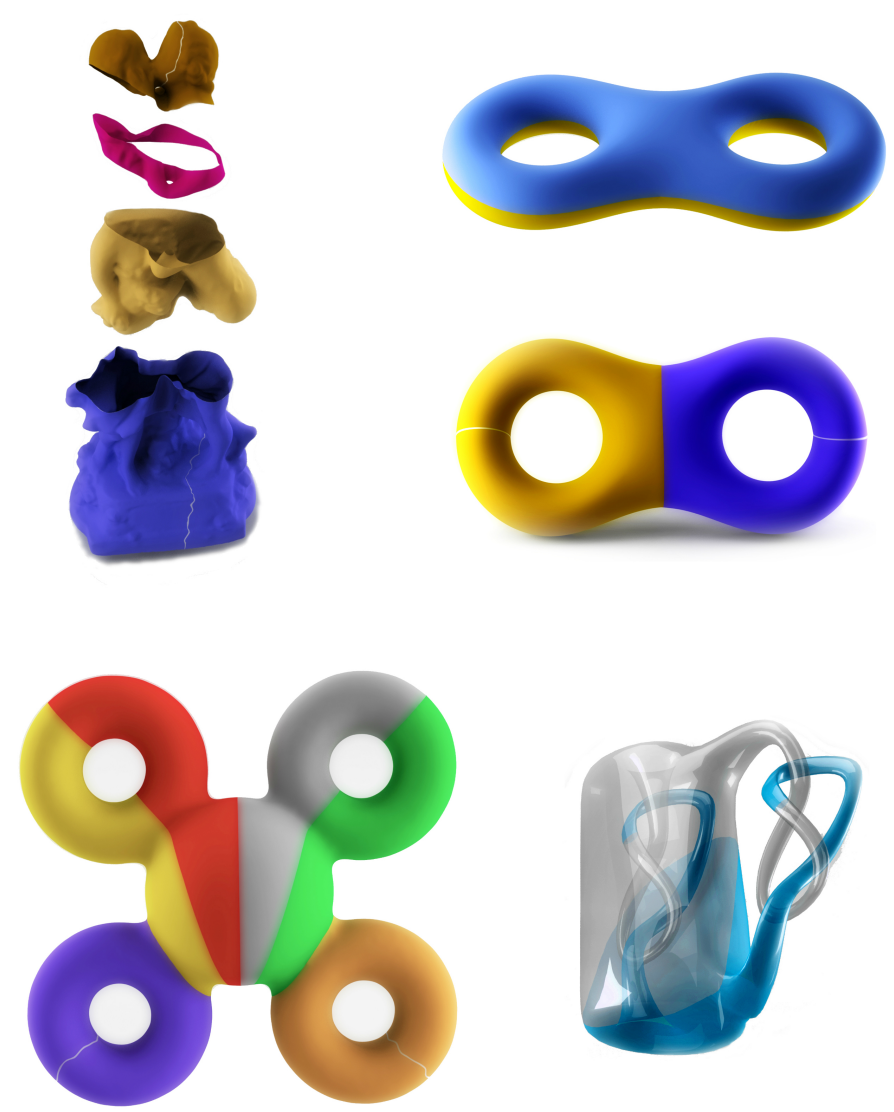

Figure 14: Pants decomposition using our Handle-based algorithm.

In the case when the Reeb graph appears as in left hand side of Figure 16 then $M_{t_{3}+\epsilon}$ is homeomorphic to a surface of type $(0, b)$ where $b \geq 4$. This surface can be decomposed into pants as we described earlier. The case when the Reeb graph appears as in the right hand side of Figure 16 then the $M_{t_{3}+\epsilon}$ is surface of type $(1, b)$ where $b>2$. We can cut this surface along the simple saddle using descending path by tracing a loop from the simple saddle point the unique minimum using descending path as we described earlier into a sphere with $b+2$ boundary components. The latter can be also decomposed into pants as explained in earlier sections. Note that this case is an extension of the simple case we considered in Lemma 4

3. The case when $p_{i}$ is a degenerate saddle where $2<i<$ $n-2$. In this case $f^{-1}\left(t_{i}-\epsilon, t_{i}-\epsilon\right)$, for a sufficiently $\epsilon$, is a finite disjoint union of multiple surfaces of type $(0,2)$ and one surface of of type $(0, b)$ where $b \geq 4$. When we have this, we attach the cylinders just as before to previous pants and we apply Morse function-based algorithm again on the single remaining sphere with $b$ boundary components to decompose it into pants.

Remark 3. We left out two cases, namely the cases when $p_{n-1}$ is degenerate and the case when $p_{n-1}$ is simple saddle and $p_{n-2}$ is a degenerate one. These two cases can be dealt with as cases (1) and (2) above. 


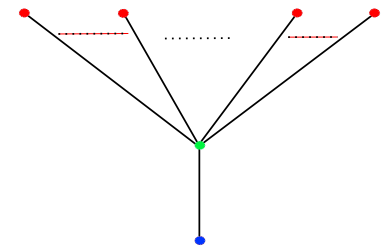

Figure 15: The Reeb graph obtained by restricting the PL scalar function on the manifold $M_{t_{2}+\epsilon}$. The red point is the unique minimum of $f$ on $M$, the green point is the degenerate saddle point, and the blue points represent the boundary circles of the surface $M_{t_{2}+\epsilon}$.
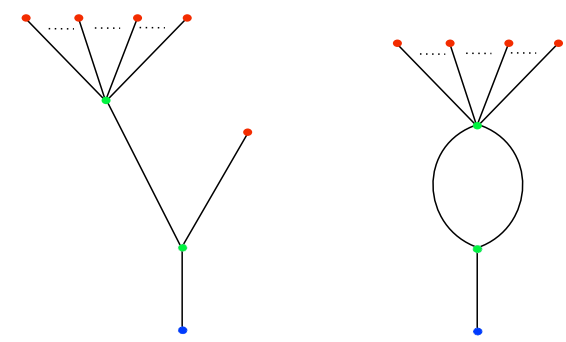

Figure 16: The two possible cases of the Reeb graph obtained by restricting the scalar function on the surface $M_{t_{3}+\epsilon}$.

\section{Reeb Graph-Based Pants Decomposition}

In this section we give pants decomposition algorithm using the Reeb graph of a Morse function. This algorithm is implicit in the work of (Hatcher, 1999). We extend this algorithm to handle degenerate cases that show up in practice. The Reeb graph decomposition algorithm has many advantages over the previous algorithm. The main advantage of this algorithm lies in the fact that it does not put any restriction on the choice of the Morse function. This allows us to choose a scalar function with better geometric properties. The second advantage is that choosing a cutting circle on a surface is much more flexible when using the structure of the Reeb graph than choosing the inverse image of a regular value of a Morse function.

Let $M$ be a compact orientable (triangulated) surface, possibly with boundary, such that $\chi(M)<0$. Let $f$ be an arbitrary (PL) Morse function of $M$. The Reeb graph-based pants decomposition algorithm of the surface $M$ and the Morse function $f$ goes as follows:

\section{Compute the Reeb graph $R(f)$ of $(M, f)$.}

2. There are two types of 1-valence nodes on the graph $R(f)$, the 1-valence nodes that are a result of collapsing the boundary components of $M$ and the 1-valence nodes that are coming from critical points of $f$ of index 0 and 2 . We consider the graph $\overline{R(f)}$ obtained by taking the deformation retract of the graph $R(f)$ that leaves the edges coming from the boundary component without retraction. This step can be done by iteratively deleting the edges one of whose nodes has valence 1 until there are no more such edges except the ones which have 1-valence nodes originating from boundary components. See Figure 17 step (3) for an example of such retraction.
3. We remove all the nodes on the graph $\overline{R(f)}$ of valency 2 and we combine the two edges that meet at such a node into one edge. We also denote the graph obtained from this step by $\overline{R(f)}$. Note that this graph is trivalent by construction.

4. We select an interior point on every edge of the graph $\overline{R(f)}$ provided that neither one of the two nodes defining that edge has valency 1 . Note that the selection of the these points on the graph $\overline{R(f)}$ corresponds to partitioning the graph $\overline{R(f)}$ into a collection of small graphs each one of them is a vertex connected by small three arcs. See step (5) in Figure 17 Each one of these small graphs corresponds to a pair of pants on the surface $M$.

5. Each choice of an interior point induces a choice of a simple closed curve on the original surface $M$. The collection of all curves obtained in this way defines a pants decomposition of the surface $M$.
(1)

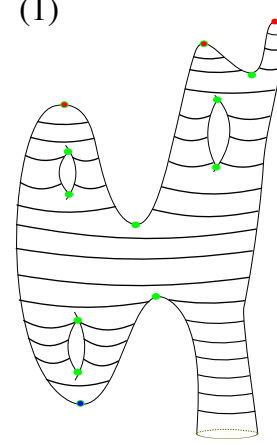

(4)

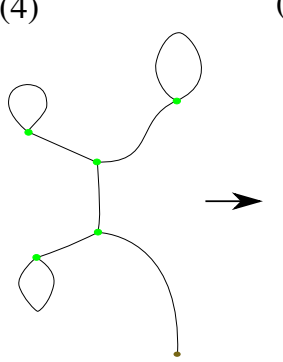

(5)
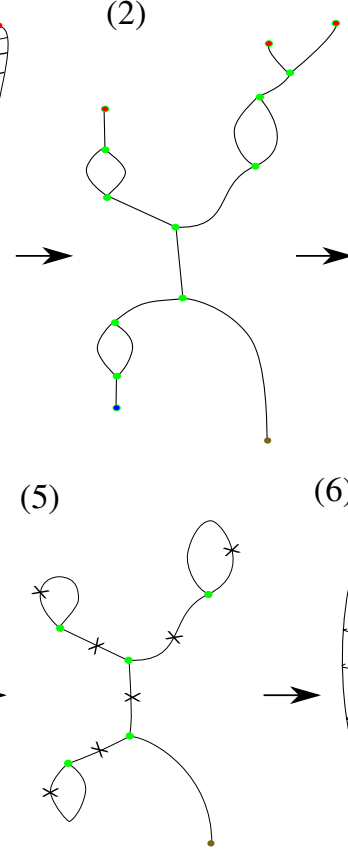

(3)

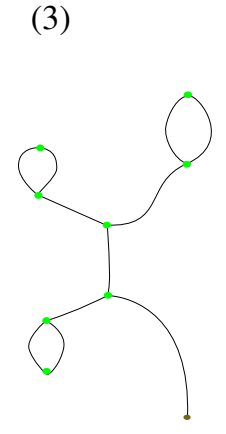

(6)

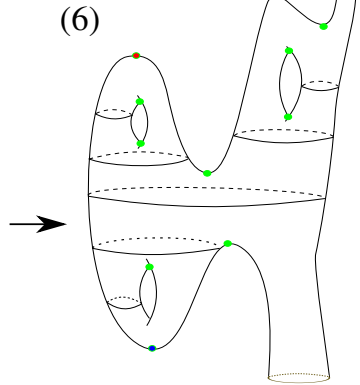

Figure 17: The steps of the Reeb graph-based pants decomposition algorithm

Figure 18 shows an application of this algorithm on some surfaces.

\subsection{Dealing with degenerate cases}

In the case when some critical points of index 1 have multiplicity $m \geq 2$, we proceed as described in steps 1 through 5 . However, the final result of the decomposition will no longer be a collection of pants but rather it will contain some surfaces of type $(0, b)$ where $b \geq 4$. For each single surface of these surfaces we apply one of the pants decomposition algorithms again in order to decompose it into a collection of pants. 


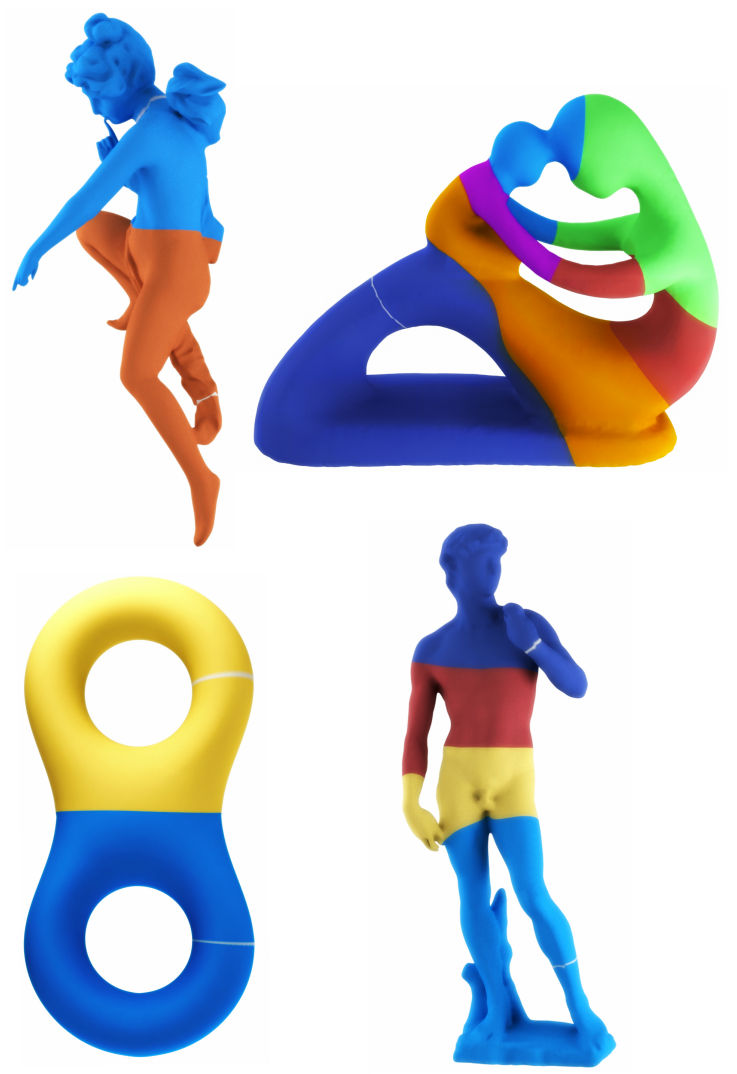

Figure 18: Pants decomposition using our Reeb graph-based algorithm

\section{Choosing an Appropriate Scalar Function}

We need to construct a function that suits the algorithms that we have presented. For the algorithm given in section 4.1 we need a scalar function that has one global minimum and one global maximum. This can be done by solving a Laplace equation on a mesh with Dirichlet boundary condition. A scalar $f$ function that satisfies the Laplace equation $\Delta f=0$ is called harmonic. We are seeking here is a scalar function $f$ which satisfies the Laplace equation $\Delta f=0$ subject to the Dirichlet boundary conditions $f\left(v_{i}\right)=c_{i}$ for all $v_{i} \in V_{C}$. Here $V_{C} \subset V$ is a set of constrained vertices and $c_{i}$ are known scalar values providing the boundary conditions. This system has a unique solution provided $\left|V_{C}\right| \geq 2$. Furthermore, the solution for such a system has an important property that it has no local extrema other than the constrained vertices. This property of harmonic functions is usually called the maximum principle (Rosenberg. 1997). Designing such a function is possible in practice. Recall that on triangulated mesh $M$ the standard discretization for the Laplacian operator at a vertex $v_{i}$ is given by :

$$
\Delta f\left(v_{i}\right)=\sum_{\left[v_{i}, v_{j}\right] \in M} w_{i j}\left(f\left(v_{j}\right)-f\left(v_{i}\right)\right),
$$

where $w_{i j}$ is a scalar weight assigned to the edge $\left[v_{i}, v_{j}\right]$ such that $\sum_{\left[v_{i}, v_{j}\right] \in M} w_{, j}=1$. Choosing the weights $w_{i j}$ such that $w_{i j}>$ 0 for all edges $\left[v_{i}, v_{j}\right.$ ] guarantees the solution of the Laplace equation has no local extrema other than at constrained vertices
$V_{C}$ (Floater, 2003). These conditions are satisfied by the mean value weights:

$$
w_{i j}=\frac{\tan \left(\theta_{i j} / 2\right)+\tan \left(\phi_{i j} / 2\right)}{\left\|v_{j}-v_{i}\right\|},
$$

where the angles $\theta_{i j}$ and $\phi_{i j}$ are the angles on either sides of the edge $\left[v_{i}, v_{j}\right]$ at the vertex $v_{i}$. Mean value weights are used to approximate harmonic map and they have the advantage that they are always non-negative which prevents any introduction of extrema on non-constrained vertices in the solution of the Laplace equation specified above. On the other hand, the cotangent weights may become negative in presence of oblique triangles and this can produce local extrema on non-constrained vertices. In this context see also (Wardetzky et al. 2007) for various discretizations of the Laplace-Beltrami operator and their properties. In our Morse function-based algorithm for pants decomposition we needed a scalar function that has precisely one global minimum and one global maximum. Hence, the constrained vertices $V_{C}$ is chosen to have exactly two vertices $V_{C}=\left\{v_{\text {min }}, v_{\text {max }}\right\}$ such that $f\left(v_{\min }\right)<f\left(v_{\max }\right)$. This choice will guarantee that the solution $f$ has a single minimum at $v_{\min }$ and a single maximum at $v_{\text {max }}$. Hence, every other critical point for $f$ must be a saddle point which is a requirement for 4.1. Similarly, a harmonic scalar function can be used to obtain the pants decomposition algorithm 4.2 for a surface with multiple boundary components.

Even though our second algorithm works on a generic Morse function, we choose a function that captures the geometry and the symmetry aspects of the mesh. This was not possible in the previous algorithm due to the restriction of the input function. Our choice for scalar functions were made based on the object itself. For organic objects like the human body we choose the Poisson field (Dong et al., 2005). On the other hand, for objects with some symmetry, we found that isometry invariant scalar functions (Sun et al. 2009; Kim et al., 2010; Wang et al., 2014) and multi-source heat kernel maps Hajij (2015) give the best results.

\section{Experiments}

\subsection{Run-time comparison}

We ran our experiments on a $3.70 \mathrm{GHz}$ AMD(R) A6-6300 with $10.0 \mathrm{~Gb}$ memory. We implement all the algorithms presented in Table 1 using $\mathrm{C}++$ on a Windows platform. The algorithms were tested on different models and compared their run-time with a publicly available software running the algorithm of (Li et al. 2009). Table 1 shows that time comparison between these algorithms. The running times that are provided in the table includes the time needed for computing the scalar functions needed as an input for in out algorithms and exclude the time needed to compute the homology generators needed for the algorithm of (Li et al. 2009). We did not include the pants decomposition algorithm presented in Zhang and $\mathrm{Li}$ (2012) since it is not different fundamentally from the one in ( $\mathrm{Li}$ et al. 2009). The main purpose of the framework 


\begin{tabular}{|c|c|c|c|c|c|}
\hline Model & Vertices & Topology & Alg. 1 time & Alg. 2 time. & Alg. Li et al., 2009) \\
\hline Eight & 3070 & $G=2$ & 0.588 & 0.322 & 11.74 \\
David & 26138 & $G=3$ & 24.637 & 5.593 & 64.43 \\
4-Torus & 10401 & $G=4$ & 4.1495 & 1.815 & 4.52 \\
Vase & 10014 & $G=2$ & 2.793 & 1.224 & 4.12 \\
Greek & 43177 & $G=4$ & 90.426 & 10.567 & 275.2 \\
Topology & 6616 & $G=13$ & 7.098 & 2.827 & Fail \\
Knotty & 5382 & $G=2$ & 1.244 & 0.606 & Fail \\
\hline
\end{tabular}

Table 1: Run-time in seconds; "Fail" means that the software crashes on the input mesh. The running time is in seconds.

in (Li et al., 2009) is to enumerate different pants decomposition classes starting from an initial pants decomposition which is computed using the method given in (Li et al., 2009). The algorithms that we presented here perform much better than the algorithm in (Li et al. 2009). In particular, the Reeb graph algorithm gives us the best time efficiency.

We mentioned in 6 that our choice of the scalar function relies on the geometry that we deal with. In Table 2 we list our choices for the scalar functions that we used in our experimentation in the Reeb graph-based pants decomposition algorithm. We also list in the same table other scalar functions that we found potentially useful and could be used on the same model to produce similar results. In our Handle-based pants decomposition algorithm all scalar fields are Harmonic fields by our remark in Section6

\begin{tabular}{|c|c|c|}
\hline Model & Our choice of the scalar field & Other appropriate scalar fields \\
\hline Eight & Laplacian Eigenfunctions & Harmonic functions, \\
David & Poisson fields & Harmonic functions \\
4-Torus & Multi-source heat kernel maps & Isometry invariant fields \\
Vase & Harmonic functions & Poisson fields \\
Greek & Harmonic functions & Poisson fields \\
Topology & Isometry invariant fields & Poisson fields \\
Knotty & Poisson fields & Harmonic functions \\
\hline
\end{tabular}

Table 2: The scalar fields used on each model in our experimentation for the Reeb graph-based algorithm.

Figure 19 shows examples of the scalar functions used in the input for our algorithms. The Figure also shows the critical points of the scalar functions.

\subsection{Imperfect input}

Just to test how robust are our algorithms against noise, we ran our algorithms on surfaces corrupted with deliberate noise. We applied random displacements to the coordinates of the input mesh and tested the results. We applied noise amplitude up to $15 \%$ of the bounding box length for the input mesh. Both algorithms maintained correct and meaningful outputs. See Figure 20 for some examples.

\section{Conclusion}

Morse theory is a powerful mathematical tool that uses local differential properties of a manifold to infer its global topological properties. Using a PL version of the theory, we have given two algorithms to decompose a surface mesh into
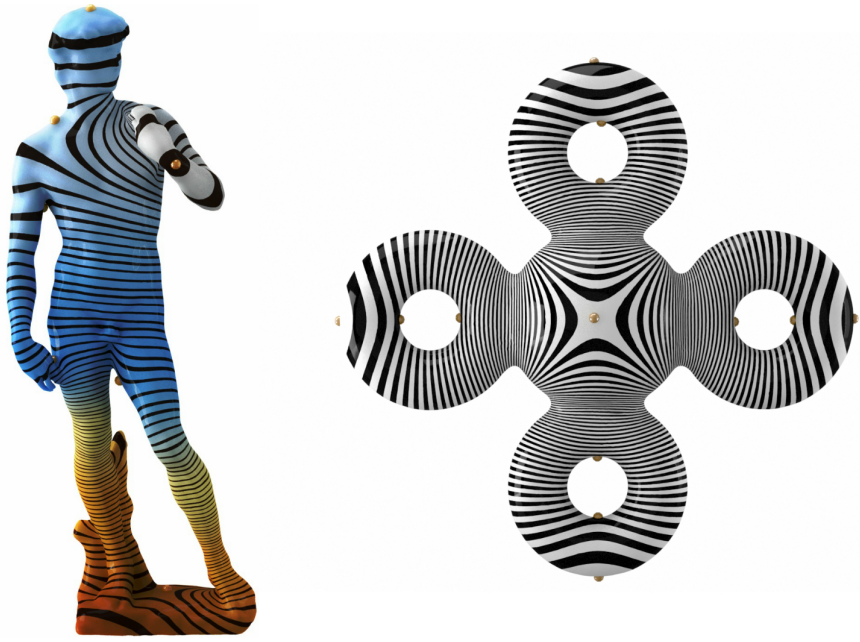

Figure 19: Two of the scalar functions used in the input for our algorithms. One the David model on the left a Poisson field is produced and on a multi-source heat kernel scalar field on the 4-Torus model on the right.
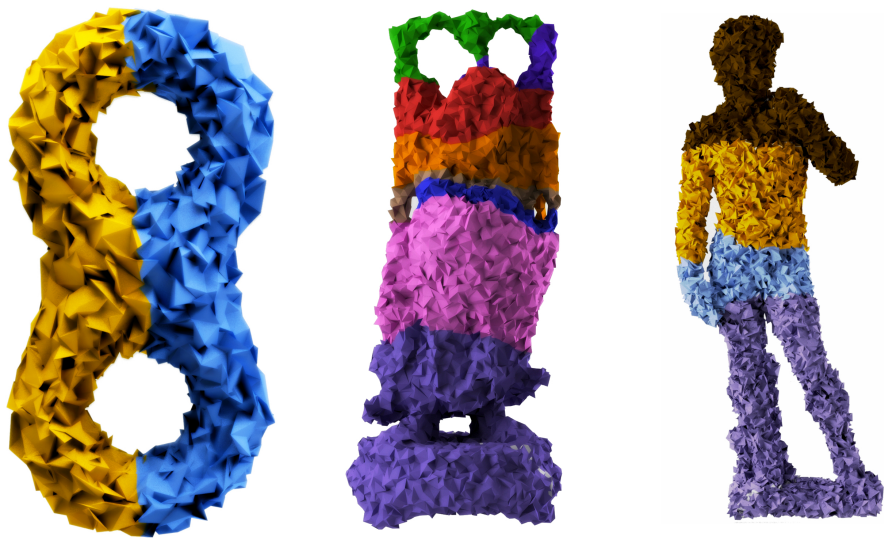

Figure 20: Pants decomposition for a noisy surface.

pants. The algorithms we proposed here remove the constrains required by earlier algorithms and the run time much faster than the existing state-of-the-art method.

The choice of a Morse function has an impact on the output of the pants decomposition algorithms that we propose here. We have proposed some scheme to compute functions suitable for our pants decomposition algorithms. These scalar functions satisfies certain desirable geometric properties. By the term desirable we mean one or more of the following properties

1. The isolines of the scalar function are shape-aware in the sense that they follow one of the principal directions of the surface.

2. The critical points of the scalar function coincide with feature or the symmetry points on the surface.

3. If the surface has some sort of symmetry then the scalar field also inherits the symmetry of the surface. 


\section{Minimal user input.}

However, are there alternative schemes which give better pants with some desirable geometric properties? We intend to address this issue in future.

\section{Acknowledgment}

This first and third authors were partly supported by NSF IIS-1320959.

Marco Attene, Silvia Biasotti, and Michela Spagnuolo. Shape understanding by contour-driven retiling. The Visual Computer, 19(2):127-138, 2003.

Marco Attene, Bianca Falcidieno, and Michela Spagnuolo. Hierarchical mesh segmentation based on fitting primitives. The Visual Computer, 22(3):181193, 2006a.

Marco Attene, Sagi Katz, Michela Mortara, Giuseppe Patané, Michela Spagnuolo, and Ayellet Tal. Mesh segmentation-a comparative study. In Shape Modeling and Applications, 2006. SMI 2006. IEEE International Conference on, pages 7-7. IEEE, $2006 \mathrm{~b}$.

Thomas Banchoff et al. Critical points and curvature for embedded polyhedra. J. Diff. Geom, 1(245-256):34, 1967.

Silvia Biasotti, Bianca Falcidieno, and Michela Spagnuolo. Extended reeb graphs for surface understanding and description. In Discrete geometry for computer imagery, pages 185-197. Springer, 2000.

Silvia Biasotti, Simone Marini, Michela Mortara, et al. An overview on properties and efficacy of topological skeletons in shape modelling. In null, page 245. IEEE, 2003.

Xiaobai Chen, Aleksey Golovinskiy, and Thomas Funkhouser. A benchmark for 3d mesh segmentation. In ACM Transactions on Graphics (TOG), volume 28, page 73. ACM, 2009.

Kree Cole-McLaughlin, Herbert Edelsbrunner, John Harer, Vijay Natarajan, and Valerio Pascucci. Loops in reeb graphs of 2-manifolds. In Proceedings of the nineteenth annual symposium on Computational geometry, pages 344-350. ACM, 2003.

Thomas M Cover and Peter E Hart. Nearest neighbor pattern classification. Information Theory, IEEE Transactions on, 13(1):21-27, 1967.

Tamal K Dey, Kuiyu Li, and Jian Sun. On computing handle and tunnel loops. In Cyberworlds, 2007. CW'07. International Conference on, pages 357366. IEEE, 2007.

Tamal K Dey, Fengtao Fan, and Yusu Wang. An efficient computation of handle and tunnel loops via reeb graphs. ACM Transactions on Graphics (TOG), 32(4):32, 2013.

Shen Dong, Scott Kircher, and Michael Garland. Harmonic functions for quadrilateral remeshing of arbitrary manifolds. Computer aided geometric design, 22(5):392-423, 2005.

Shen Dong, Peer-Timo Bremer, Michael Garland, Valerio Pascucci, and John C Hart. Spectral surface quadrangulation. In ACM Transactions on Graphics (TOG), volume 25, pages 1057-1066. ACM, 2006.

Harish Doraiswamy and Vijay Natarajan. Efficient algorithms for computing reeb graphs. Computational Geometry, 42(6):606-616, 2009.

Michael S Floater. Mean value coordinates. Computer aided geometric design, 20(1):19-27, 2003.

Xiaohu Guo, Xin Li, Yunfan Bao, Xianfeng Gu, and Hong Qin. Meshless thinshell simulation based on global conformal parameterization. Visualization and Computer Graphics, IEEE Transactions on, 12(3):375-385, 2006.

Mustafa Hajij. Constructing desirable scalar fields for morse analysis on meshes. 2015.

W. Harvey, R. Wenger, and Y. Wang. A randomized $O(m \log m)$ time algorithm for computing Reeb graph of arbitrary simplicial complexes. In Proc. 25th Annu. ACM Sympos. Compu. Geom., pages 267-276, 2010.

Allen Hatcher. Pants decompositions of surfaces. arXiv preprint math/9906084, 1999.

Allen Hatcher and William Thurston. A presentation for the mapping class group of a closed orientable surface. Topology, 19(3):221-237, 1980.

Franck Hétroy and Dominique Attali. Topological quadrangulations of closed triangulated surfaces using the reeb graph. Graphical Models, 65(1):131$148,2003$.
Masaki Hilaga, Yoshihisa Shinagawa, Taku Kohmura, and Tosiyasu L Kunii. Topology matching for fully automatic similarity estimation of $3 \mathrm{~d}$ shapes. In Proceedings of the 28th annual conference on Computer graphics and interactive techniques, pages 203-212. ACM, 2001.

Miao Jin, Wei Zeng, Ning Ding, and Xianfeng Gu. Computing fenchel-nielsen coordinates in teichmuller shape space. In Shape Modeling and Applications, 2009. SMI 2009. IEEE International Conference on, pages 193-200. IEEE, 2009.

PIZZANU Kanongchaiyos and YOSHIHISA Shinagawa. Articulated reeb graphs for interactive skeleton animation. Proceeding Modeling Multimedia Information and System, pages 451-467, 2000.

George Karypis and Vipin Kumar. A software package for partitioning unstructured graphs, partitioning meshes, and computing fill-reducing orderings of sparse matrices. University of Minnesota, Department of Computer Science and Engineering, Army HPC Research Center, Minneapolis, MN, 1998.

Vladimir G Kim, Yaron Lipman, Xiaobai Chen, and Thomas Funkhouser. Möbius transformations for global intrinsic symmetry analysis. In Computer Graphics Forum, volume 29, pages 1689-1700. Wiley Online Library, 2010.

Tsz-Ho Kwok, Yunbo Zhang, and Charlie CL Wang. Constructing common base domain by cues from voronoi diagram. Graphical Models, 74(4):152$163,2012$.

Bruno Lévy, Sylvain Petitjean, Nicolas Ray, and Jérome Maillot. Least squares conformal maps for automatic texture atlas generation. In ACM Transactions on Graphics (TOG), volume 21, pages 362-371. ACM, 2002.

Xin Li, Xianfeng Gu, and Hong Qin. Surface mapping using consistent pants decomposition. Visualization and Computer Graphics, IEEE Transactions on, 15(4):558-571, 2009.

Xin Li, Wuyi Yu, and Celong Liu. Geometry-aware partitioning of complex domains for parallel quad meshing. Computer-Aided Design, 2016.

Xuetao Li, Tong Wing Woon, Tiow Seng Tan, and Zhiyong Huang. Decomposing polygon meshes for interactive applications. In Proceedings of the 2001 symposium on Interactive $3 D$ graphics, pages 35-42. ACM, 2001.

Alan P Mangan and Ross T Whitaker. Partitioning $3 \mathrm{~d}$ surface meshes using watershed segmentation. Visualization and Computer Graphics, IEEE Transactions on, 5(4):308-321, 1999.

Yukio Matsumoto. An introduction to Morse theory, volume 208. American Mathematical Soc., 2002.

John Willard Milnor. Morse theory. Number 51. Princeton university press, 1963.

Xinlai Ni, Michael Garland, and John C Hart. Fair morse functions for extracting the topological structure of a surface mesh. ACM Transactions on Graphics (TOG), 23(3):613-622, 2004

Salman Parsa. A deterministic $O(m \log m)$ time algorithm for the Reeb graph. In ACM Sympos. Comput. Geom. (SoCG), pages 269-276, 2012.

Valerio Pascucci, Giorgio Scorzelli, Peer-Timo Bremer, and Ajith Mascarenhas. Robust on-line computation of reeb graphs: simplicity and speed. In $A C M$ Transactions on Graphics (TOG), volume 26, page 58. ACM, 2007.

Giuseppe Patane, Michela Spagnuolo, and Bianca Falcidieno. Para-graph: Graph-based parameterization of triangle meshes with arbitrary genus. In Computer Graphics Forum, volume 23, pages 783-797. Wiley Online Library, 2004.

Georges Reeb. Sur les points singuliers dune forme de pfaff completement intgrable ou dune fonction numrique. CR Acad. Sci. Paris, 222:847-849, 1946.

Douglas Reynolds, Richard C Rose, et al. Robust text-independent speaker identification using gaussian mixture speaker models. Speech and Audio Processing, IEEE Transactions on, 3(1):72-83, 1995.

Steven Rosenberg. The Laplacian on a Riemannian manifold: an introduction to analysis on manifolds. Number 31. Cambridge University Press, 1997.

Hiroshi Sato, Takahisa Takino, Yasunori Okada, Jian Cao, Akira Shinagawa, Etsuhide Yamamoto, and Motoharu Seiki. A matrix metalloproteinase expressed on the surface of invasive tumour cells. Nature, 370(6484):61-65, 1994.

Ariel Shamir. A survey on mesh segmentation techniques. In Computer graphics forum, volume 27, pages 1539-1556. Wiley Online Library, 2008.

Yoshihisa Shinagawa and Tosiyasu L Kunii. Constructing a reeb graph automatically from cross sections. IEEE Computer Graphics and Applications, (6):44-51, 1991.

Shymon Shlafman, Ayellet Tal, and Sagi Katz. Metamorphosis of polyhedral surfaces using decomposition. In Computer Graphics Forum, volume 21, 
pages 219-228. Wiley Online Library, 2002.

Barton T Stander and John C Hart. Guaranteeing the topology of an implicit surface polygonization for interactive modeling. In Proceedings of the 24th annual conference on Computer graphics and interactive techniques, pages 279-286. ACM Press/Addison-Wesley Publishing Co., 1997.

Jian Sun, Maks Ovsjanikov, and Leonidas Guibas. A concise and provably informative multi-scale signature based on heat diffusion. In Computer graphics forum, volume 28, pages 1383-1392. Wiley Online Library, 2009.

Carlo Tomasi and Roberto Manduchi. Bilateral filtering for gray and color images. In Computer Vision, 1998. Sixth International Conference on, pages 839-846. IEEE, 1998.

Hongyu Wang, Ying He, Xin Li, Xianfeng Gu, and Hong Qin. Geometry-aware domain decomposition for t-spline-based manifold modeling. Computers $\mathcal{E}$ Graphics, 33(3):359-368, 2009.

Hui Wang, Patricio Simari, Zhixun Su, and Hao Zhang. Spectral global intrinsic symmetry invariant functions. In Proceedings of the 2014 Graphics Interface Conference, pages 209-215. Canadian Information Processing Society, 2014.

Max Wardetzky, Saurabh Mathur, Felix Kälberer, and Eitan Grinspun. Discrete laplace operators: no free lunch. In Symposium on Geometry processing, pages 33-37, 2007.

Naoufel Werghi, Yijun Xiao, and Jan Paul Siebert. A functional-based segmentation of human body scans in arbitrary postures. Systems, Man, and Cybernetics, Part B: Cybernetics, IEEE Transactions on, 36(1):153-165, 2006.

Ichitaro Yamazaki, Vijay Natarajan, Zhaojun Bai, and Bernd Hamann. Segmenting point sets. In Shape Modeling and Applications, 2006. SMI 2006. IEEE International Conference on, pages 6-6. IEEE, 2006.

Wuyi Yu and Xin Li. A geometry-aware data partitioning algorithm for parallel quad mesh generation on large-scale 2d regions. Procedia Engineering, 124: 44-56, 2015.

Eugene Zhang, Konstantin Mischaikow, and Greg Turk. Feature-based surface parameterization and texture mapping. ACM Transactions on Graphics (TOG), 24(1):1-27, 2005.

Kang Zhang and Xin Li. Optimizing geometry-aware pants decomposition. In Proc. Pacific Graphics, pages 11-16, 2012.

Emanoil Zuckerberger, Ayellet Tal, and Shymon Shlafman. Polyhedral surface decomposition with applications. Computers $\mathcal{E}$ Graphics, 26(5):733-743, 2002. 